\title{
Double-Difference Waveform Inversion of 4D Ocean Bottom Cable Data: Application to Valhall, North Sea
}

\author{
Di Yang*, Michael Fehler and Alison Malcolm, MIT, Faqi Liu and Scott Morton, Hess Corporation
}

\section{SUMMARY}

Changes in reservoir properties resulting from extracting hydrocarbons and injecting fluid are critical to optimize production. These properties can be characterized using waveform inversions of time-lapse seismic data. The conventional approach for analysis using waveform tomography is to take the difference of seismic inversion obtained using baseline and subsequent time-lapse datasets that are imaged independently. By contrast, double-difference waveform inversion (DDWI) jointly inverts time-lapse seismic datasets for reservoir changes. We use a $2 \mathrm{D}$ synthetic example to demonstrate the advantage of DDWI in mitigating spurious estimates of property changes. We then apply both conventional full waveform inversion(FWI) and DDWI to time-lapse datasets collected by ocean bottom cables (OBC) in the Valhall field in the North Sea. The data sets are acquired one year apart. DDWI gives a cleaner and more easily interpreted image of the model changes, as compared to that obtained with the conventional FWI scheme.

\section{INTRODUCTION}

Time-lapse seismic monitoring is widely used in reservoir management in the oil industry to obtain information about reservoir changes caused by fluid injection and subsequent production. The seismic responses change according to the fluid saturations and pressures in the reservoir. The optimal goal of time-lapse seismic is to track fluid flow in areas without well logs (Lumley, 2001). During the time interval of production, the geologic structures are assumed to be time-invariant. Thus, differences between images formed with multiple timelapse datasets are assumed to show changes due to fluid flow. Conventional analysis of time-lapse seismic data only gives either qualitative information like seismic amplitude or indirect quantitative parameters like image shifts and traveltime differences. This information needs to be transferred to reservoir properties by matching reservoir modeling (Lumley and Behrens, 1998).

Waveform inversion has the potential to estimate subsurface density and elasticity parameters quantitatively (Tarantola, 1984), and it has become feasible with recent increases in computing power. Ideally, by subtracting the models inverted from each data set in a series of time-lapse surveys, the geophysical property changes over time can be quantified. Watanabe et al. (2005) applied a differential waveform tomography in the frequency domain for crosswell time-lapse data during gas production and showed that the results are more accurate for estimating velocity changes in small regions than those obtained using the conventional method. Onishi et al. (2009) also applied a similar strategy to conduct differential traveltime tomography using crosswell surveys. Denli and Huang
(2009) developed a double-difference waveform tomography algorithm using time-lapse reflection data in the time domain and demonstrated using synthetic data that the method has the potential to produce reliable estimates of reservoir changes.

In this work, a 2D synthetic example is used to demonstrate how DDWI can improve the inversion quality in terms of suppressing spurious model perturbations. We then apply this strategy to the 4D OBC field data in an oil field in the North Sea. By inverting the time-lapse data that are one year apart, the resulting model reveals a zone with increased velocity under the gas cloud. We compare our results with that obtained from a conventional inversion scheme, and show that DDWI produces a cleaner and more interpretable image of the reservoir change.

\section{THEORY}

The algorithm of DDWI consists of two parts. The first one is a traditional waveform inversion of the data from a baseline survey using an earth model obtained from standard model building technologies, e.g., tomography. The waveform inversion minimizes a cost or objective function of the difference between modeled data and baseline data:

$$
E(m)=\frac{1}{2}\left|u_{\text {baseline }}-d_{\text {baseline }}\right|^{2}=\frac{1}{2} \delta u^{T} \delta u,
$$

where $u_{\text {baseline }}$ and $d_{\text {baseline }}$ are the displacements of forward modeling and baseline data, respectively, $\delta u=u_{\text {baseline }}-d_{\text {baseline }}$, the superscript ${ }^{T}$ denotes the transpose, and $m$ is the parameter (P-wave velocity) to be updated. Gradient-based methods are generally used to solve such waveform inversion problems, resulting in a local minimum.

After the inversion of the baseline data, the inverted model is used as the initial model in the DDWI for time-lapse seismic data. DDWI is very similar to the inversion scheme described above, with a modification of $\delta u$ in the cost function (Equation.1) as

$$
\delta u=\left(u_{\text {lapse }}-u_{\text {baseline }}\right)-\left(d_{\text {lapse }}-d_{\text {baseline }}\right),
$$

where $u_{\text {baseline }}$ and $u_{\text {lapse }}$ are synthetics from the starting model obtained from inversion of the baseline data and the model to be updated, and $d_{\text {baseline }}$ and $d_{\text {lapse }}$ are baseline and time-lapse data, , respectively. DDWI inverts for the change in the model that causes the waveform changes between time-lapse datasets. It can reduce the effects of uncertainties in the initial model.

The first derivative of the cost function (Equation 3) with respect to the time-lapse residual $\left(u_{\text {lapse }}-d_{\text {lapse }}\right)$ is zero when the two residuals are identical, leading to a minimum of the function. In other words, the model difference is cleaned by matching the two inversions' local minima.

$$
\frac{\partial E(m)}{\partial\left(u_{\text {lapse }}-d_{\text {lapse }}\right)}=\left(u_{\text {lapse }}-d_{\text {lapse }}\right)
$$




\section{Double-difference waveform inversion of 4D Ocean Bottom Cable Data}

$$
-\left(u_{\text {baseline }}-d_{\text {baseline }}\right)
$$

\section{MARMOUSI MODEL}

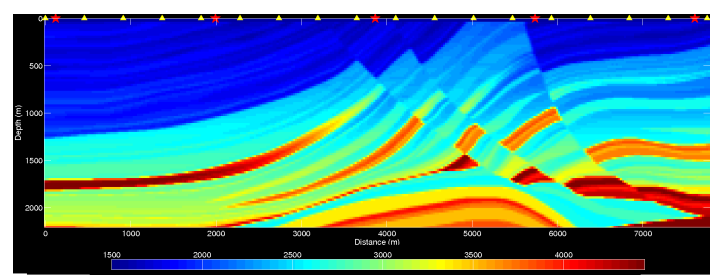

Figure 1: True P-wave velocity baseline model. The colorbar denotes velocity values.

In this section, we use the Marmousi model to illustrate the different behaviors of standard FWI and DDWI. Figure 1 is the true baseline earth model. We use five shots marked as red stars in Figure 1 that are evenly spaced near the water surface and 350 receivers (yellow triangles) at the same depth as sources. We generate synthetic acoustic data which are used as the baseline data.

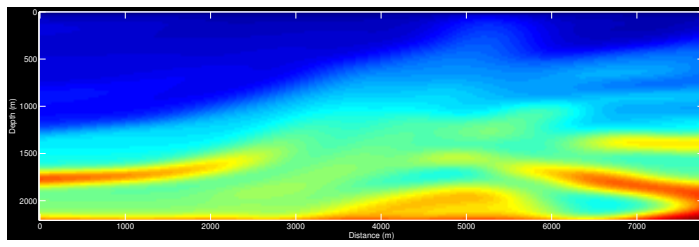

(a)

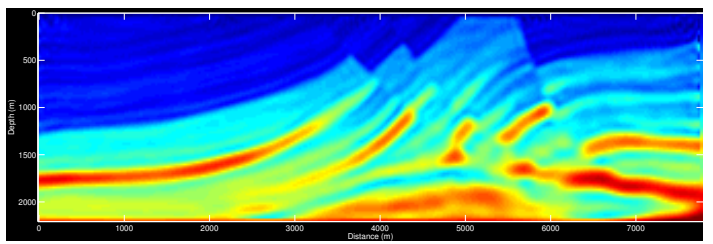

(b)

Figure 2: (a) Starting velocity model for FWI. (b) The velocity model obtained after 80 iterations. Colorscales are the same as Figure 1.

We use a smoothed version of the Marmousi model (Figure 2(a)) as a starting model for the standard FWI and employ the conjugate gradient method to invert for the P-wave velocity model.

After 80 iterations, we obtain the recovered baseline model shown in Figure 2(b). It contains the dominant features of the structures and velocity distributions, while some of the finer and deeper parts of the model are not well-recovered.

We simulate the injection by a decrease in velocity in a triangular region marked with the arrow in Figure 3.

Starting from the model in Figure 2(b), there are two ways to invert for the model change. One is to directly run FWI to fit time-lapse data based on the baseline model. The other is to apply DDWI to fit the seismic signal difference based on

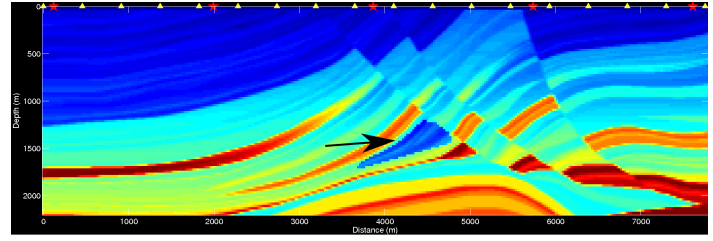

Figure 3: True P-wave velocity time-lapse model with the same colorscale as Figure(1)

the same model. The model changes inverted using these two approaches are shown in Figures 4(a) and 4(b) respectively.

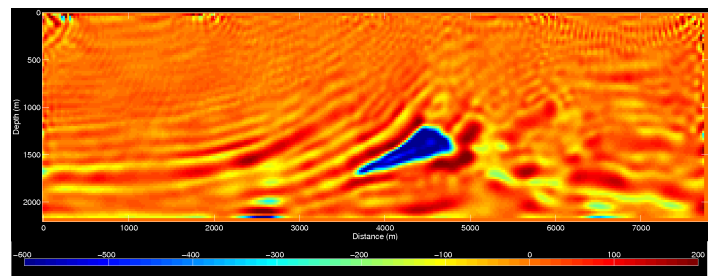

(a)

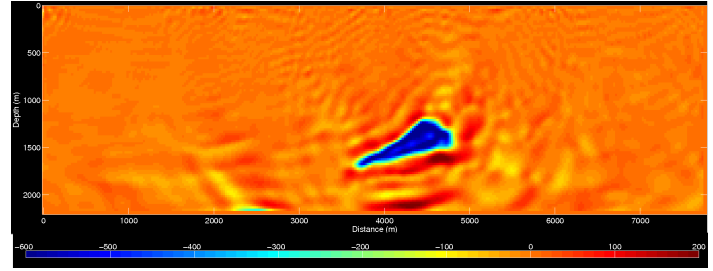

(b)

Figure 4: Time-lapse velocity change inverted by FWI (a), and DDWI (b).

In the direct FWI result, the velocity anomaly is recovered well both in shape and amplitude. However, outside the area of interest, there are model perturbations almost everywhere following the background structures as indicated in Figure 1. The amplitude of the undesired structures are relatively low, mainly because the synthetic data are inverted up to $95 \%$ of their energy in an L-2 norm measure. The data residuals compared to the actual seismic signal changes are small. Accordingly, the model residuals are small compared to the reservoir changes.

The DDWI result (Figure 4(b)) recovers the anomaly, as the conventional FWI does, but the background update is much weaker, and less misleading because it is not following the geology structures. The non-reservoir-related updates here are actually very small in amplitudes. We boost the image up to better reveal the difference.

\section{VALHALL LIFE-OF-FIELD SEISMIC}

We test both the traditional FWI and DDWI on a 4D dataset in the Valhall field in the North Sea. The field has been producing for a long time, and various water injection projects are taking place. The ocean bottom cable (Barkved et al., 2003) was installed for explorational survey and long term monitoring; 1-3 


\section{Double-difference waveform inversion of 4D Ocean Bottom Cable Data}

repeat surveys are conducted every year. Each survey involves about 2.5 thousand permanently emplaced receivers and tens of thousands of shots on the water surface. The entire survey covers an area of $15 \mathrm{~km} \times 8 \mathrm{~km}$.

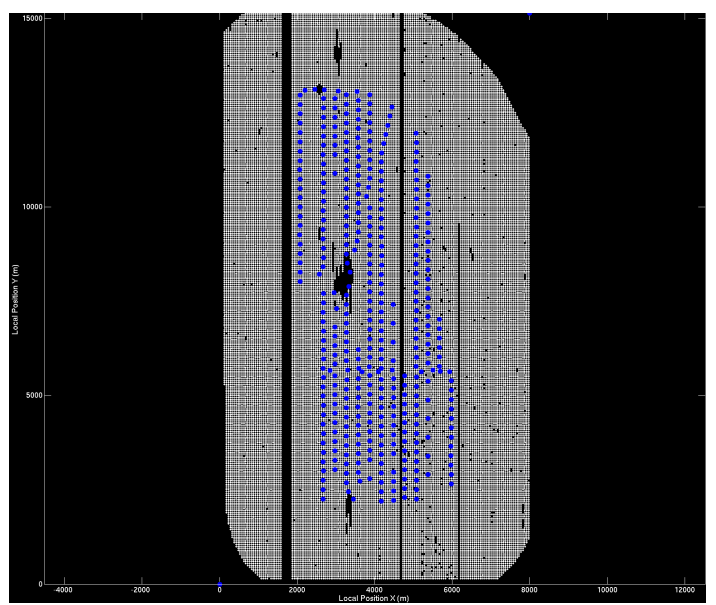

Figure 5: Locations of the sources and receivers used in this work.

To make the application computationally more practical, we only use 379 receivers and all the shots in the surveys (Figure 5). The baseline survey and time-lapse survey are one year apart. Shots were positioned, within a small deviation $(<5 \mathrm{~m})$, on a regular grid. Since finite difference modeling requires the shots to be on regular grids, we use the method in Hicks (2002) to interpolate the shots of both surveys to the same regular locations.

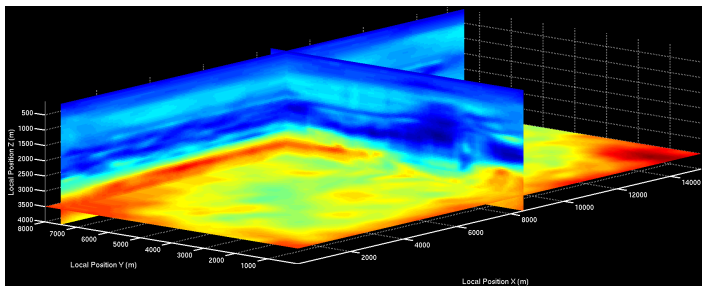

Figure 6: Initial model for FWI.

\section{Inversion Setup}

A few assumptions are made in this inversion process. First, only the pressure data are used in this work, and so the acoustic wave equation is solved to simulate the wavefield. Second, only the P-wave velocity is inverted. The density model is derived from the Gardner Equation (Gardner et al., 1974) using the updated velocity model in each iteration. Third, attenuation and anisotropy are not included in the modeling.

\section{Initial Velocity Model}

The baseline inversion is a complicated process in practice (Liu et al., 2012). It is difficult to use only FWI to invert for a model with good quality starting from a poor initial guess. In this study, we use a smoothed version of the velocity presented in Liu et al. (2012) as shown in Figure 6, to avoid the elaborate process of model building, since this study focuses on the time-lapse application.

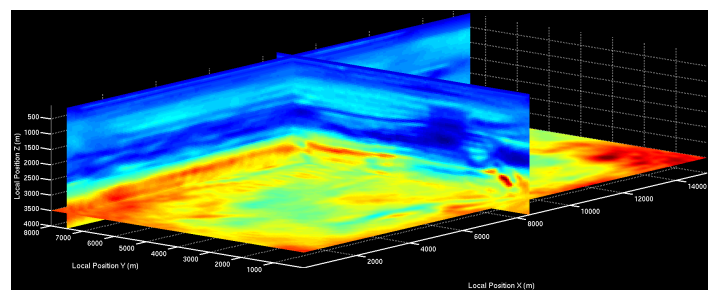

Figure 7: Baseline model obtained after 200 iterations.

\section{Baseline Inversion Result}

We run acoustic FWI for the baseline survey data starting from the model in Figure 6. After 200 iterations, the baseline model is considered to converge as shown in Figure 7 . The final model is of higher resolution than the input model, the image of the gas cloud (y-z slice in Figure 7) is markedly improved, and the thin layer under the gas cloud is well resolved.

\section{Timelapse Inversion Result}

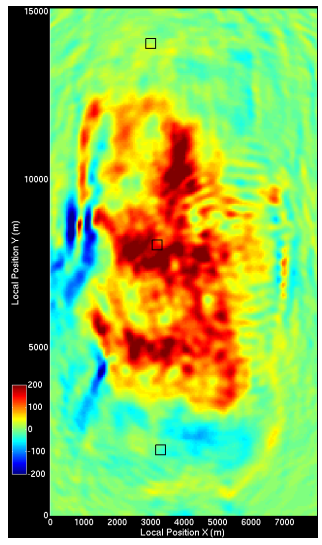

(a)

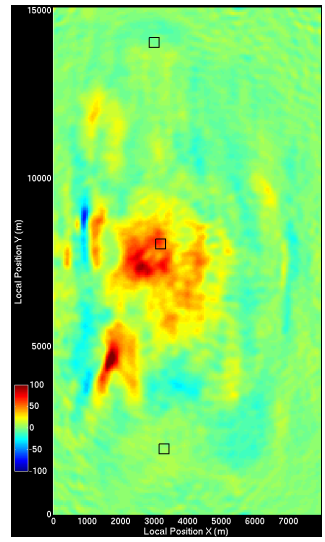

(b)
Figure 8: X-Y slice at the depth where maximum time-lapse changes occur. (a) Time-lapse changes resolved by standard FWI; (b) Time-lapse changes resolved by DDWI.

Starting from the model in Figure 7, we run the regular FWI with the time-lapse dataset and also apply DDWI. To better compare the results between the two inversion schemes, we plot the 2D slices instead of the whole volume in Figures 8 and 9. Depth slices at the location of the maximum timelapse velocity changes are shown in Figure 8. The three black squares mark the holes in the survey(Figure 5). The time-lapse change from standard FWI(Fig 8(a)) is of larger amplitude and covers a broader area than the result from DDWI(Fig 8(b)). The change from DDWI is localized around the center of the area.

The two Y-Z cross-sectional views in Figure 9 show the timelapse change in velocity for standard FWI and DDWI. The model changes have completely different patterns. In the traditional FWI case, the velocity change spreads over most of the area in the deeper part of the model, and some distinct 


\section{Double-difference waveform inversion of 4D Ocean Bottom Cable Data}

changes are also found in the shallow parts. By contrast, in the DDWI case, the dominant change is localized in the center of the model beneath the gas cloud. The changes in other parts are much weaker, and no evident changes are found in the shallow part of the model.

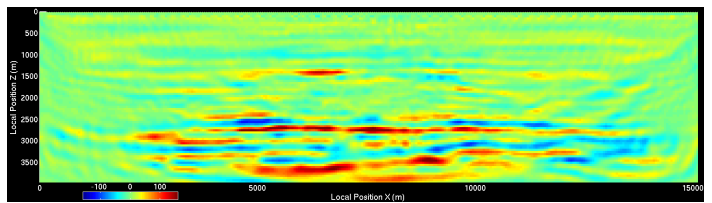

(a)

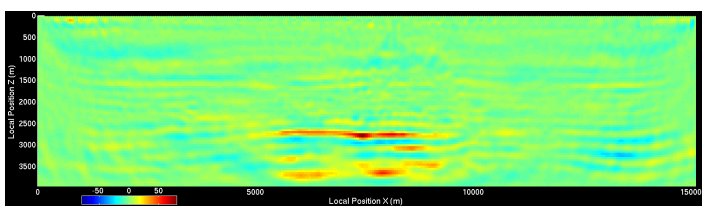

(b)

Figure 9: Y-Z slice of the velocity change at the location where maximum time-lapse changes occur along the $\mathrm{X}$-axis. (a) Time-lapse changes resolved by standard FWI; (b) Timelapse changes resolved by DDWI.

\section{DISCUSSION}

In this work we observed that the difference between traditional FWI and DDWI results are larger in amplitude and bigger in extent in field data than in synthetics. According to the derivation in the theory, the difference is caused by how the two methods treat the data residuals. Unlike the synthetic case in which the baseline data are fit up to a very high level, the fitting in the real baseline data application is limited because of issues such as incomplete physics, imprecise source signature and noise contamination. In addition, the actual change we set in the synthetic study is $600 \mathrm{~m} / \mathrm{s}$, which is much larger than the strongest change we obtain in the field application. The contrast between the background model residual and the time-lapse change is smaller in the real data case. For standard FWI, a better simulator with more physics included should improve the data fitting in the real data case, and also suppress the strong model changes that are not 4-D effects as in Figure 9(a).

If we take the field data results at face-value, DDWI is definitely finding a time-lapse velocity change that is cleaner and easier to interpret. But to understand why this is the case, and thus to increase our confidence in our interpretation, we first need to describe what we are fitting in DDWI and how this varies with traditional FWI. To this end, Figure 10 summarizes the various effects that we expect to see in the data and highlights those that are supressed with DDWI with colors as compared with standard FWI. DDWI as described in the theory section, is trying to fit the difference between baseline and time-lapse data to invert for the corresponding model perturbation. The data can be decomposed into two parts as shown in Figure 10: signal and noise. In the noise branch, we classify noise as either coherent or random. The random compo-

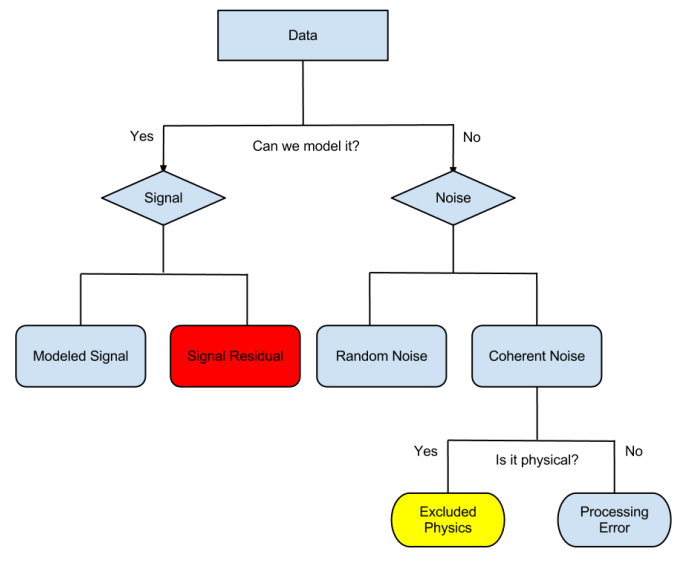

Figure 10: The decomposition of the data.

nent will contribute relatively little to the final image because of stacking. Coherent noise should lead to changes throughout the model, if it is constructively interfering and significant enough. In DDWI, the common part of coherent noise between two datasets is subtracted out. The signal that is not modeled due to incomplete physics (Yellow in Figure 10) in the model equations has a second-order effect on the velocity change. For example, the common background anisotropy and attenuation effects are subtracted out in DDWI, and those induced by reservoir change are relatively weak and localized. Within the signal branch, the residual signal that can be modeled (Red in Figure10), and due to either under-fitting the data or being caught in a local minimum is what we expect to cancel in DDWI and not in FWI.

Because the model change in the DDWI example is clean and localized, it is credible that the recovered velocity change is actually the reservoir change rather than simply the movement into a different local minimum of the objective function, or simply the change one might expect if the inversion were to be continued to additional iterations.

To further verify the results, we would apply DDWI on more datasets to see if the 4-D effects are consistent and compare our 4-D differences to those obtained by standard 4-D imaging. Applications to Valhall life-of-field seismic datasets that are farther apart in time would be expected to show similar but stronger 4-D changes. Moreover, more physics should be included in the modeling such as anisotropy and elasticity in our future work.

\section{ACKNOWLEDGMENTS}

The authors want to thank Hess Corporation for supporting this study and providing computing facilities. We also thank Hess and BP for providing this data. We are also grateful to Lianjie Huang from Los Alamos National Lab for constructive comments and discussions. MIT Earth Resources Lab Consortia members are also acknowledged for sponsoring the research. 
http://dx.doi.org/10.1190/segam2013-1318.1

\section{EDITED REFERENCES}

Note: This reference list is a copy-edited version of the reference list submitted by the author. Reference lists for the 2013 SEG Technical Program Expanded Abstracts have been copy edited so that references provided with the online metadata for each paper will achieve a high degree of linking to cited sources that appear on the Web.

\section{REFERENCES}

Barkved, O., A. Baerheim, D. Howe, J. Kommedal, and G. Nicol, 2003, Life of field seismic implementation: First at Valhall: Presented at the $65^{\text {th }}$ Annual InternationalConference and Exhibition, EAGE, Extended Abstracts, A.

Denli, H., and L. Huang, 2009, Double-difference elastic waveform tomography in the time domain: $79^{\text {th }}$ Annual International Meeting, SEG, Expanded Abstracts, 28, 2302-2306.

Gardner, G., L. Gardner, and A. Gregory, 1974, Formation velocity and density - The diagnostic basics for stratigraphic traps: Geophysics, 39, 770-780, http://dx.doi.org/10.1190/1.1440465.

Hicks, G. J., 2002, Arbitrary source and receiver positioning in finite-difference schemes using Kaiser windowed sinc functions: Geophysics, 67, 156-166.

Liu, F., L. Guasch, S. A. Morton, M. Warner, A. Umpleby, Z. Meng, S. Fairhead, and S. Checkles, 2012, 3D time-domain full waveform inversion of a Valhall OBC data set: $82^{\text {nd }}$ Annual Internaitonal Meeting, SEG, Expanded Abstracts, doi:10.1190/segam2012-1105.1.

Lumley, D., and R. Behrens, 1998, Practical issues of 4D seismic reservoir monitoring: What an engineer needs to know: SPE Reservoir Evaluation \& Engineering, 1, no. 6, 528-538, http://dx.doi.org/10.2118/53004-PA.

Lumley, D. E., 2001, Time-lapse seismic reservoir monitoring: Geophysics, 66, 50-53, http://dx.doi.org/10.1190/1.1444921.

Onishi, K., T. Ueyama, T. Matsuoka, D. Nobuoka, H. Saito, H. Azuma, and Z. Xue, 2009, Application of crosswell seismic tomography using difference analysis with data normalization to monitor $\mathrm{CO}_{2}$ flooding in an aquifer: International Journal of Greenhouse Gas Control, 3, no. 3, 311-321, http://dx.doi.org/10.1016/j.ijggc.2008.08.003.

Tarantola, A., 1984, Inversion of seismic reflection data in the acoustic approximation: Geophysics, 49 , 1259-1266, http://dx.doi.org/10.1190/1.1441754.

Watanabe, T., S. Shimizu, E. Asakawa, and T. Matsuoka, 2005, Differential waveform tomography for time-lapse crosswell seismic data with application to gas hydrate production monitoring: Presented at the $75^{\text {th }}$ Annual International Meeting, SEG. 